\title{
Central motor conduction in a family with hereditary motor and sensory neuropathy with pyramidal signs (HMSN V)
}

\author{
Armin Schnider, Christian W Hess, Stefan Koppi
}

\begin{abstract}
Two generations of a family affected by hereditary motor and sensory neuropathy with pyramidal signs (HMSN V) were clinically and electrophysiologically examined. Apart from electroneurographic studies, the central motor conduction (CMC) to arm and leg muscles was assessed using magnetic transcranial motor cortex stimulation. Abnormal CMC was confined to the clinically affected members, with the exception of an unaffected subject who had a diminished but normal latency response in a leg. The typical pattern was a significant diminution of the compound muscle action potential from the tibialis anterior and a moderately prolonged cortico-muscular conduction time (CoMCT) to this muscle.
\end{abstract}

Inherited peroneal muscular wasting and sensory disturbances in the legs with a stocking like distribution are found in a variety of disorders summarised by Dyck ${ }^{1}$ as hereditary motor and sensory neuropathy (HMSN). The disease described here is a rare variant within this group, which is associated with signs of a central motor pathway lesion, that is, with positive pyramidal signs and relatively brisk tendon reflexes. It has been referred to as hereditary motor and sensory neuropathy type V (HMSN V) by Dyck ${ }^{1}$ or peroneal muscular atrophy with pyramidal features by Harding et al. $^{2}$ Most cases have autosomal dominant inheritance. The onset of symptoms and signs is in the second decade or later. ${ }^{1}$ The disease usually progresses slowly so that the patients remain ambulant and do not get severely handicapped.

Electrophysiological investigations have revealed few changes of motor and sensory nerve conduction. The velocity and amplitude of the sural nerve were slightly diminished or low normal ${ }^{3}$ and the compound motor action potentials decreased in amplitude. The EMG of affected muscles show signs of denervation. In sural biopsies, there is loss of large myelinated fibres with a virtual absence of both segmental demyelination and onion-bulb formation. ${ }^{4}$ These electrophysiological and histological findings are consistent with primary axonal nerve degeneration and closely resemble the changes found in the neuronal type of HMSN, so called HMSN type II.

Little is known about the involvement of central motor pathways in HMSN V. The introduction of magnetic brain stimulation in clinical medicine ${ }^{5}$ provides a sensitive and harmless tool for assessing central motor conduction (CMC) in humans. In this study, we have measured the CMC to arm and leg muscles on both sides in two generations of a family with HMSN V, of which some young healthy members are very likely to carry the disease. It was our aim to describe the changes typical of the CMC in HMSN V and to find out whether subclinical involvement could be detected with the method.

\section{Patients and methods}

All 19 members of generations II and III of the family (fig 1) were willing to participate in the study and gave their informed consent. For the deceased parents of the offspring (generation I), a meticulous family history were collected. We did not examine members of the fourth generation, as we do not perform magnetic brain stimulation for scientific purposes in subjects younger than 16 years.

The family members were considered clinically affected if weakness of the lower limbs, peroneal muscle wasting with pes cavus, and signs of upper motor neuron involvement, that is, extensor plantar responses, were present. Brisk tendon jerks alone were not interpreted as evidence of the disease. The subjects were classified as possibly affected if only symptoms or signs of polyneuropathy were found, such as polymodal sensory disturbances of typical distribution or diminished ankle jerks without peroneal muscle wasting.

In all the clinically affected members, nerve conduction studies and, in four of them, electromyographic studies were performed using a DISA Electromyograph 1500. Orthodromic sural neurography was carried out with surface stimulation at the lateral malleolus and unipolar needle recording $15 \mathrm{~cm}$ proximally, following the Behse and Buchthal method. ${ }^{6}$ Peroneal neurography was carried out with surface recordings from the extensor digitorum brevis muscle and surface stimulation. Electromyography was performed with conventional concentric needle electrodes (bandpass 2 to $10000 \mathrm{~Hz}$ ). In three patients, the mean duration of at least 20 motor unit potentials was calculated for quantitative assessment. ${ }^{7}$

Central motor conduction time (CMCT) to the upper limbs was assessed by magnetic 
Figure 1 Distribution of the disease in the family examined. The arrow indicates a family member with a cerebellar syndrome of unknown origin.

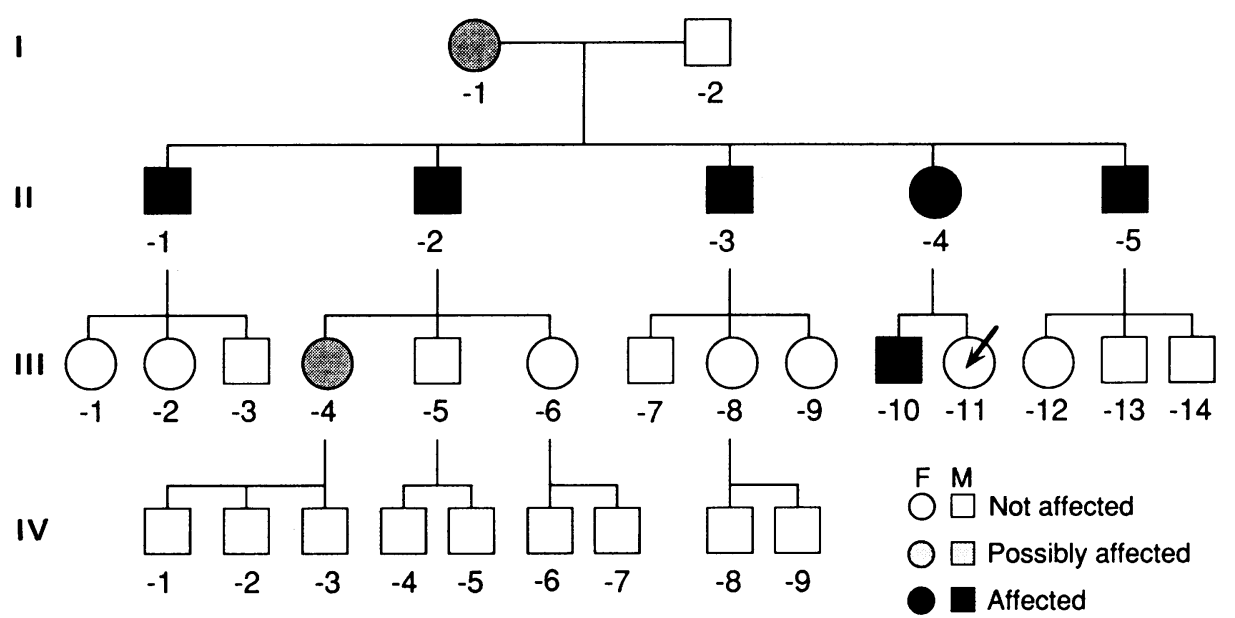

stimulation of the motor cortex and high voltage electrical stimulation of the cervical motor root as described earlier. ${ }^{58}$ For motor cortex stimulation a specially designed powerful magnetic siimulator with a total capacitance of $800 \mu \mathrm{F}$ and a total maximal charging energy of 2500 Joules was employed. $^{9}$ A circular stimulating coil of a mean diameter of $8 \mathrm{~cm}$ and a total inductance of $30 \mu \mathrm{H}$ was placed over the vertex with the current (positive flux) flowing anti-clockwise as viewed from above to excite the right limbs and vice versa. ${ }^{5}$ For assessing central motor conduction to the lower limb, the centre of the stimulating coil was centred 2 to $4 \mathrm{~cm}$ anterior to the vertex. When it proved difficult to get an unequivocal response in the leg muscle, a more powerful 8-shaped twin coil with a total inductance of $33 \mu \mathrm{H}$ was used. This coil produces a deep, focused stimulus and invariably elicits easily recognisable responses in the tibialis anterior muscles of healthy subjects. ${ }^{9}$ The shortest of at least three collected responses was taken for latency and the largest for amplitude evaluation. Cervical stimulation was performed using transcutaneous high voltage discharges (DIGITIMER D 180) with the cathode over the C7/T1 interspace and the anode $6 \mathrm{~cm}$ cranially on the midline. Surface recordings on both sides from the abductor digiti minimi muscles (ADM), biceps brachii muscles (BICEPS), and tibialis anterior muscles (TA) were collected. To facilitate the responses, the subjects exerted a slight, steady voluntary contraction during cortical stimulation. The responses were recorded on a MEDELEC Sensor 94a system interfaced to a microcomputer.

CMCT to the ADM and BICEPS was defined as the latency difference between the onset of the compound muscle action potential (CMAP) from cortical and cervical stimulation. For the TA the total cortex to muscle conduction time (CoMCT) was taken in relation to the subject's height. In one affected subject (III-10) where the CoMCT was clearly prolonged, the lumbosacral roots were additionally stimulated to obtain a peripheral conduction time and calculate a CMCT. The same high voltage technique as for the cervical root was used in this case with the cathode over the L5/S1 interspace and the anode $9 \mathrm{~cm}$ cranially. Normal values in our laboratory are as follows: upper limit of normal defined as the mean $+2.5 \times$ standard deviations for the ADM CMCT $\leq 8.6 \mathrm{~ms}$, for the BICEPS CMCT $\leq 8.4 \mathrm{~ms}$, for the TA CoMCT $\leq(0.32 \times \mathrm{cm}$ height $)-22.48 \mathrm{~ms}$ or CMCT $\leq(0.19 \times \mathrm{cm}$ height $)-14.81 \mathrm{~ms}$. Lower limits of normal CMAP amplitude for the $\mathrm{ADM}$ is $15 \%$ of that from wrist stimulation and for the $\mathrm{TA} \geq 1.2 \mathrm{mV}$.

Statistical group analysis was carried out with the Mann-Whitney $U$ test for nonparametric data.

\section{Results}

Symptoms and signs of the examined affected

Table Clinical findings in the affected members, the possibly affected member III-4 and the subject III-11 with a cerebellar syndrome of unknown origin

\begin{tabular}{|c|c|c|c|c|c|c|c|c|c|c|c|c|}
\hline \multirow{2}{*}{$\begin{array}{l}\text { Subject } \\
\text { number }\end{array}$} & \multirow[b]{2}{*}{ Sex } & \multirow{2}{*}{$\begin{array}{l}\text { Age } \\
\text { (years) }\end{array}$} & \multicolumn{2}{|c|}{ Onset of symptoms ${ }^{1}$} & \multirow{2}{*}{$\begin{array}{l}\text { Weakness } \\
\text { of legs }\end{array}$} & \multicolumn{2}{|c|}{ Sensory loss } & \multirow[b]{2}{*}{ Atrophy ${ }^{2}$} & \multicolumn{3}{|c|}{ Reflexes $^{3}$} & \multirow{2}{*}{$\begin{array}{l}\text { Plantar } \\
\text { response }\end{array}$} \\
\hline & & & Hist & Subj & & Arms & Legs & & Arms & $K J$ & $A J$ & \\
\hline II-1 & $\mathbf{M}$ & 67 & 20 & 45 & ++ & - & ++ & $\mathrm{Pm}$ and $\mathrm{pg}$ & 2 & 2 & 0 & extensor \\
\hline II -2 & M & 66 & 20 & 55 & ++ & - & ++ & Pm and pg & 2 & 1 & 0 & extensor \\
\hline II -3 & M & 63 & 19 & 61 & ++ & + & ++ & $\mathrm{Pm}$ and $\mathrm{pg}$ & 2 & 2 & 0 & extensor \\
\hline II -4 & $\mathrm{~F}$ & 63 & 40 & 40 & ++ & + & ++ & $\mathrm{Pm}$ and pg & 2 & 2 & 0 & extensor \\
\hline II $-5^{4}$ & $M$ & 56 & $?$ & $?$ & ++ & - & + & $\mathrm{Pm}$ and pg & 1 & 1 & $1^{4 a}$ & extensor \\
\hline III-4 & $F$ & 41 & - & - & - & - & + & Pes c & 2 & 1 & 1 & flexor \\
\hline III-10 & $\mathbf{M}$ & 38 & 37 & 37 & + & - & + & $\mathrm{Pm}$ & 2 & 2 & 2 & extensor \\
\hline III-1I & $\mathrm{F}$ & 32 & 1 & 4 & - & - & + & Pes c & 1 & 1 & 1 & extensor \\
\hline
\end{tabular}

'Hist $=$ First symptoms shown by history; Subj = Start of subjectively felt handicap

${ }^{2}$ Pes $\mathrm{c}=$ Pes cavus only; $\mathrm{Pm}=$ Peroneal muscles; $\mathrm{pg}=$ Pelvic girdle.

${ }_{3}^{3}$ Grading of reflexes: $0=$ absent, $1=$ normal, $2=$ brisk; $\mathrm{KJ}=$ Knee jerk; $\mathrm{AJ}=$ Ankle jerk.

${ }^{4}$ Poliomyelitis, mainly involving right leg, at the age of 13 months.

ta $\mathrm{AJ}$ missing on the right side. 

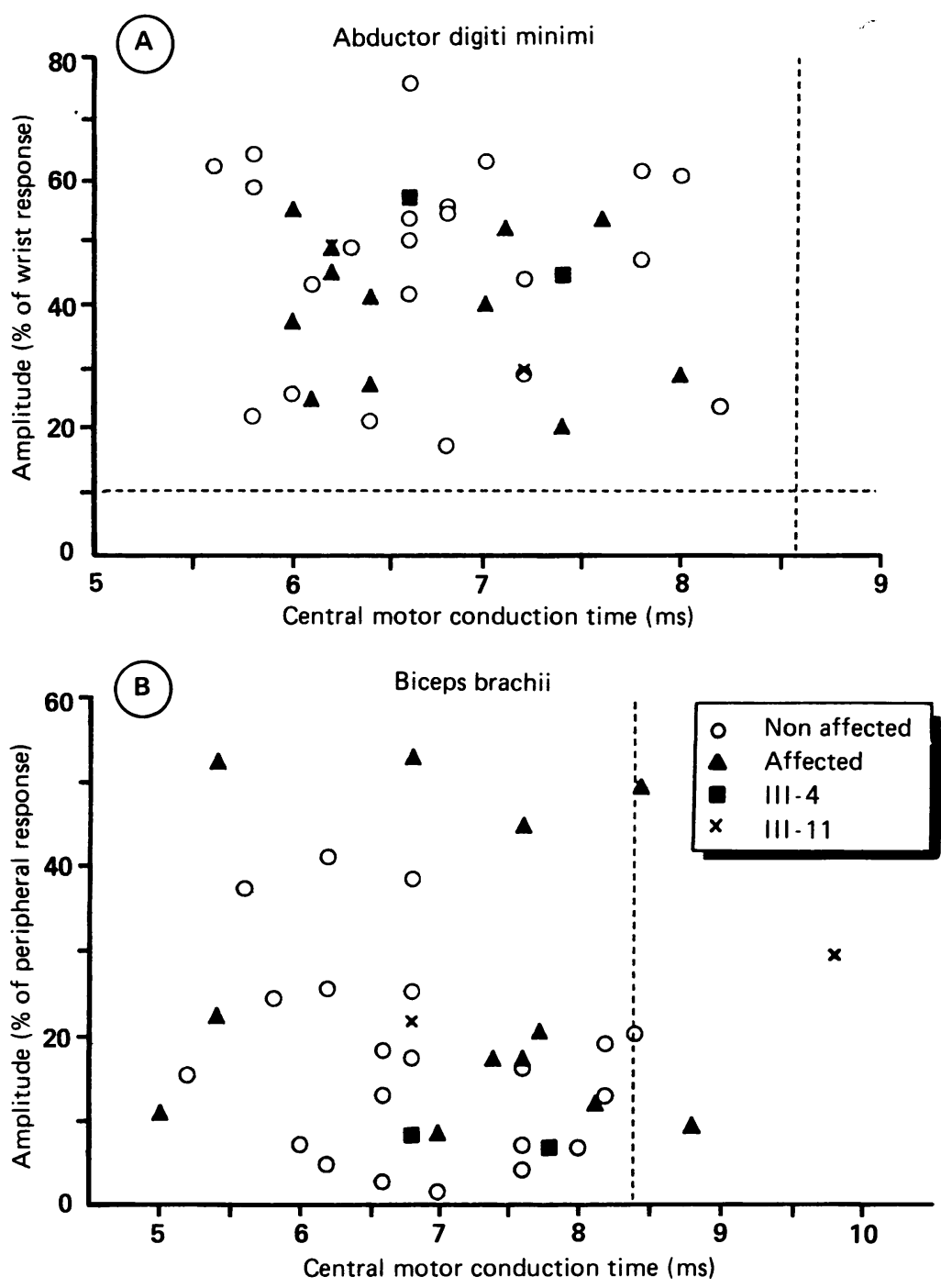

Figure 2 Central motor conduction to the abductor digiti minimi muscle (a) and to the biceps brachii muscle (b). Upper and lower limits of normal are indicated by the dashed lines (mean, $2.5 \times S D$ ). Open circles represent values from non-affected, triangles from affected members, and squares with a central point from the possibly affected member. Member III-11 (cross) had a cerebellar syndrome of early onset.

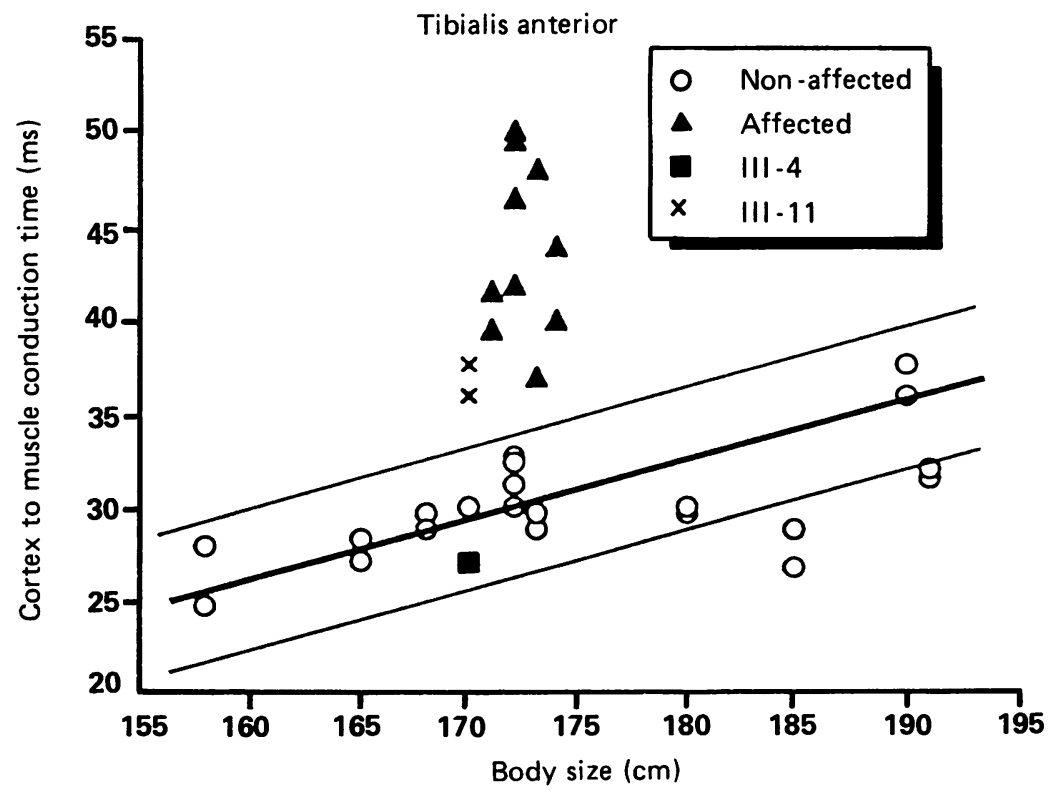

Figure 3 Cortico-muscular conduction time to the tibialis anterior muscle in relation to the subjects' height. Normal range is shown by the thin lines (mean, $2.5 \times S D)$. In one member (II-4) no measurable potential could be obtained (not included in the graph). Same symbols as in fig 2. and possibly affected family members are summarised in the table. The deceased mother I-1 was considered possibly affected since she was described by her children as having had the same sort of thin calves and broad-based gait as they had. All five members of generation II presented with peroneal muscular wasting with pyramidal signs. In generation III, only one subject (III-10) had convincing clinical evidence of the disease. Another member (III-4) had slight sensory disturbances of the legs, which she was unaware of. She was considered possibly affected. One member (III-11) presented with a cerebellar syndrome of unknown origin. She was born prematurely and could walk only from the age of $\mathbf{3 0}$ months. At four years, tremor of the arms and the head and limb ataxia developed, which were slowly progressive. The disease resembled early onset cerebellar ataxia with retained tendon reflexes as described by Harding ${ }^{10}$ with the exception that visual evoked potentials were delayed and that no evidence of inheritance was found. A mild tremor was observed in all affected members, in three non-affected members, and in subject III-11.

Various gait disturbances were found. All affected members had some foot drop, and all presented with gait ataxia which considerably worsened with the eyes closed. Half of them also showed a spastic component. The sensory disturbances were always of a stocking and glove-like distribution and concerned all sensory qualities. Those not affected were between 19 and 40 years old.

\section{Central motor conduction studies}

Arms CMCT to the ADM was normal in all instances (fig 2a). The mean (SD) of the CMCT was $6.70(0.69) \mathrm{ms}$ in the affected group and $6.72(0.75) \mathrm{ms}$ in the non-affected group (subjects III-4 and III-11 excluded).

CMCT to the biceps (fig 2b) was clearly prolonged $(9.8 \mathrm{~ms})$ on one side in patient III11 with the cerebellar syndrome, and slightly prolonged on one side in the affected members II-3 and II-4 $(8.44 \mathrm{~ms}$ and $8.80 \mathrm{~ms})$. The possibly affected member III-4 had normal CMCTs to the ADM (6.6 ms and $7.4 \mathrm{~ms}$ on the right and left side, respectively) and to the biceps $(7.8 \mathrm{~ms}$ and $6.8 \mathrm{~ms})$. Among the nonaffected members, one (III-3) had a CMCT of upper limit $(8.4 \mathrm{~ms})$. The mean (SD) of the CMCT to the biceps was $7 \cdot 11(1 \cdot 24) \mathrm{ms}$ in the affected group and $6.82(0.91) \mathrm{ms}$ in the nonaffected group (subjects III-4 and III-11 excluded). This difference was not statistically significant $(\mathrm{p}=0.35)$.

Legs In all clinically non-affected subjects, the CoMCT to the TA was normal (fig 3). With one exception the amplitude was always greater than $1.2 \mathrm{mV}$. In the clinically normal subject III-5 the TA response on the left side was diminished in amplitude with a normal latency.

All affected subjects showed both distinctly prolonged CoMCTs as well as diminished amplitudes (fig 4). Amplitudes were sometimes reduced to the extent of hardly being recognisable (II-2), and in patient II-4 no potential was obtained. In patient III-10 stimulation of 

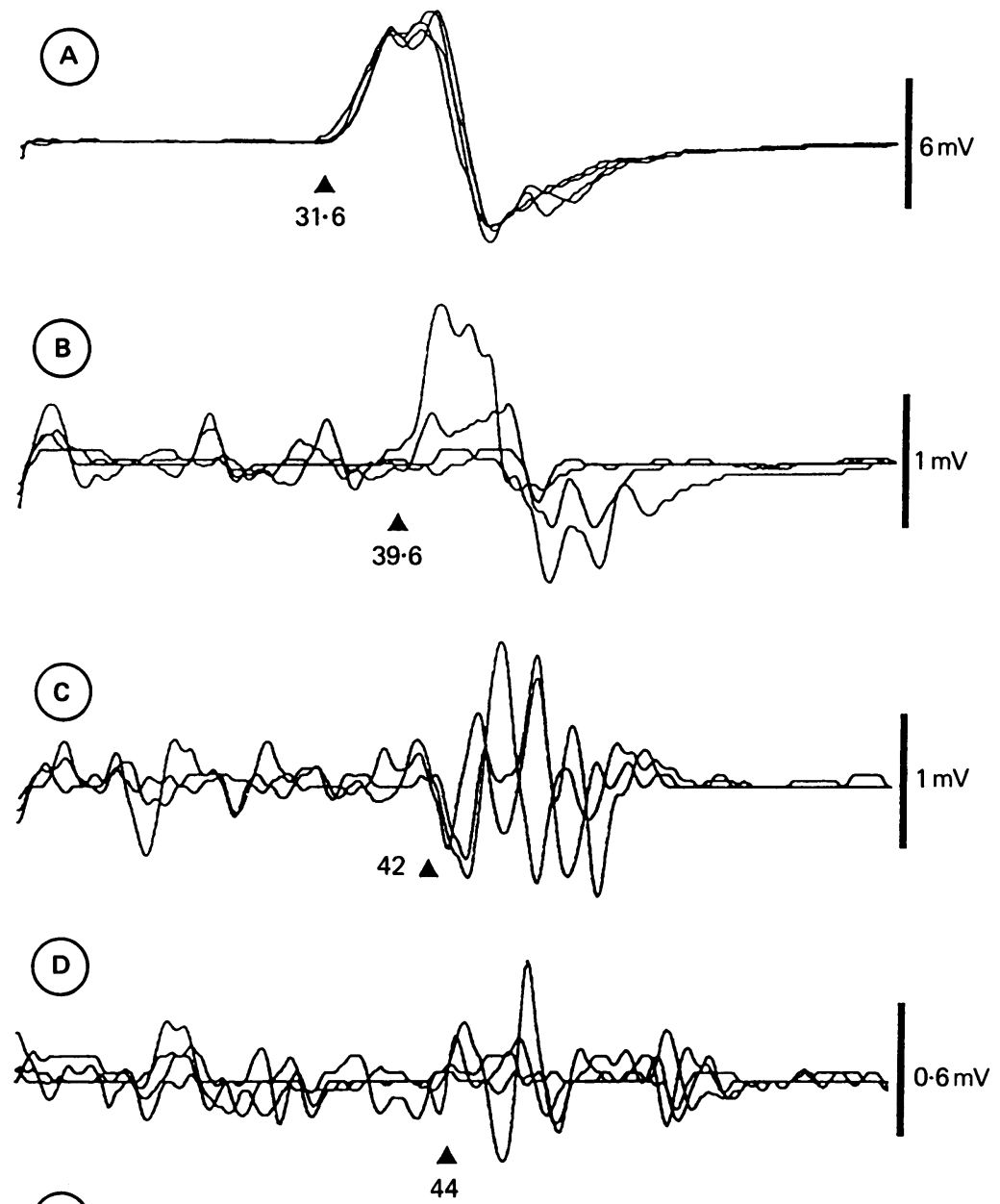

(E)

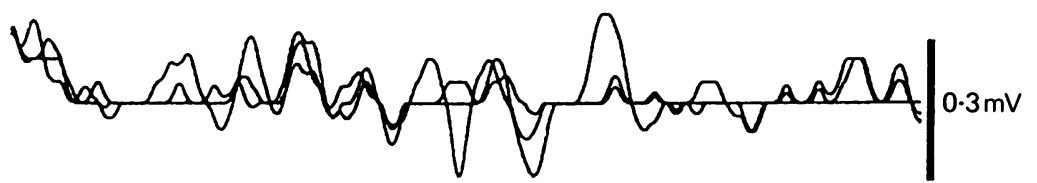

$20 \mathrm{~ms}$

Figure 4 Examples of responses from the tibialis anterior muscle following magnetic brain stimulation. Three responses are superimposed in each. Normal responses (a) from a non affected member (III-7), abnormal responses from (b) the youngest affected member (III-10), and (c-e) from three affected members of the second generation $c$ : $I I-1, d: I I-2$, and $d: I I-4$, the latter without recognisable response.

the lumbosacral roots at the L5/S1 interspace ruled out a peripheral slowing causing the cortex-muscle delay. The peripheral latency was normal and the calculated CMCT hence prolonged.

In the affected group the mean CoMCT and amplitude to magnetic brain stimulation was $43.82(4.50) \mathrm{ms}$ and $1.06(0.24) \mathrm{mV}$ respectively, and in the non-affected group this was $30 \cdot 18(2 \cdot 85) \mathrm{ms}$ and $4.37(2 \cdot 43) \mathrm{mV}$. These differences were statistically significant (CoMCT, $\mathrm{p}=0.0001 ;$ Amplitude, $\mathrm{p}=0.0002$ ).

\section{Neurography}

In all but one (II-3) of the six affected members, the sensory nerve conduction of the sural nerve was assessed. The conduction velocity was low normal in all instances. In addition, the sensory nerve action potential was abnormally split (more than five phases) in two cases (II-2, II-4), and of abnormally low amplitude in a further case (II-5). In one affected member the nerve potential was normally shaped (II-1). In member III-4 who was considered "possibly affected", sural neurography yielded an abnormal splitting of the sensory nerve action potential without slowing of conduction velocity, and in member III-11 who presented with a cerebellar syndrome, sural neurography was entirely normal.

Motor neurography on peroneal or tibial nerve yielded a conduction velocity of lower limit or completely normal in the affected members II-3 and III-10, respectively, while in patient II-1 no CMAP was obtained from an atrophic extensor digitorum brevis muscle.

\section{Electromyography}

Quantitative needle myography which was performed in three patients (II-1: TA, II-2 and II4: rectus femoris) showed neurogenic changes with moderate prolongation of the mean potential duration and, in the case of the TA, a loss of motor units. Sparse spontaneous activity was found only in the latter muscle. In patient II-3, electromyography of several leg muscles was neurogenic.

\section{Discussion}

A strikingly close correlation between assessed $\mathrm{CMC}$ and clinical findings was found in this study of a family suffering from HMSN V. Not only was CMC abnormal in all clinically affected members and normal in the clinically nonaffected members, but the abnormality was almost entirely confined to recordings from the weak leg muscles, while CMC to the upper limbs, where no atrophy or weakness was prominent, was mostly normal. Since the peripheral sensory and motor nerve conduction velocities as assessed in several affected members of the family typically were normal or only scarcely reduced, the delayed and diminished responses in the TA from cortical stimulation most likely reflect the pathology in the central motor pathways. This suggestion is further supported by the additional lumbosacral root stimulation performed in one patient with clearly delayed "central" responses. Lumbosacral stimulation yielded a normal peripheral conduction time to the TA and thus a clearly increased CMCT as calculated from the "central" and "peripheral" latencies.

Several studies have established the value of assessing CMC in the early diagnosis of multiple sclerosis (MS), ${ }^{811}$ motor neuron disease, ${ }^{1213}$ and degenerative ataxic disorders, ${ }^{14}$ where the method has proved a very sensitive and easy to perform procedure. In the HMSN, slightly abnormal CMCT to the ADM was also reported in cases with positive pyramidal signs ${ }^{15} 16$ some of them belonging to the group of HMSN V. These authors did not study the CMC to a leg muscle. The scarcity of abnormal CMCs in the third generation of our family was rather surprising. In view of the dominant inheritance 
and the fact that all members of the second generation bear the disease, some subclinically affected descendants are very likely to have it. But some members have only just reached the age of manifestation as reported in the literature. ${ }^{12}$ Even the one member who was possibly affected as judged from clinical examination (III-4 in fig 1), did not have an abnormal CMC to the upper or lower limbs. Moreover, CMC to the biceps was mostly normal in the affected members even in the presence of brisk arm reflexes. It seems therefore that measurement of CMC is not capable of revealing subclinical or slight central motor involvement in the disease. This is in contrast to what has been found in MS, where clinically silent lesions of central motor pathways have been repeatedly found by this method and where abnormal CMC findings to limbs with increased tendon reflexes is the rule. ${ }^{811}$

Furthermore, unlike the findings in MS, but similar to the results in motor neuron disease, amplitude reduction was the prominent feature of abnormality and paralleled the latency increase in all cases. To obtain a response from the TA in the clinically affected members of the family, it was necessary to use cortical stimulation with a greater output energy than that available from commercial stimulators, and with a more powerful eight-shaped twin coil. In one affected member, no response was obtained from the TA. Absent responses from motor cortex stimulation is a rare finding in MS, but is more typical of motor neuron disease, ${ }^{1213}$ where axonal loss in the pyramidal system is known to occur. Although amplitude reduction together with prolongation is also a frequent finding in MS, clearly delayed responses with normal amplitude are encountered in half the cases of MS. ${ }^{8}$ The discrepancies between the CMC findings of distinct diseases might be due to the different nature of the lesions in the central nervous system. To our knowledge, there are no necropsy reports on central nervous pathology in HMSN V. Behse and Buchthal, however, found that loss of large myelinated fibres is the prominent abnormality in the sural nerves in two of five cases stated to have "hereditary spastic paraplegia with peroneal muscular atrophy".4 The similarity between the CMC findings in our patients and in motor neuron disease suggests that in HMSN V, the lesion of the central motor pathways is also predominantly axonal rather than demyelinating.

We thank Dr D Eggenschwiler and Ms J Schmid-Sigron for their help. This study was supported by the Swiss National Science Foundation No. 3.852-0.86 and the Swiss MS Society.

1 Dyck PK. Inherited neuronal degeneration and atrophy affecting peripheral motor, sensory, and autonomic neurons. In: Dyck PJ, Thomas PK, Lambert EH, Bunge R, eds. Peripheral neuropathy, Vol II, 2nd ed. Philadelphia: WB Saunders, 1984:1600-55.

2 Harding AE, Thomas PK. Peroneal muscular atrophy with pyramidal features. J Neurol Neurosurg Psychiatry 1984 47:168-72.

3 Buchthal F, Behse F. Peroneal muscular atrophy (PMA) and related disorders. I. Clinical manifestations as related to biopsy findings, nerve conduction and electromyography. Brain 1977;100:41-66.

4 Behse F, Buchthal F. Peroneal muscular atrophy (PMA) and related disorders. II. Histological findings in sural nerves. Brain 1977;100:67-85.

5 Hess CW, Mills KR, Murray NMF. Responses in small hand muscles from magnetic stimulation of the human brain. J Physiol 1987;388:397-419.

6 Behse F, Buchthal F. Normal sensory conduction in the nerves of the leg in man. J Neurol Neurosurg Psychiatry 1971;34:404-14.

7 Ludin HP. Electromyography in practice. Stuttgart: Thieme, 1980.

8 Hess CW, Mills KR, Murray NMF, Schriefer TN. Magnetic brain stimulation: central motor conduction studies in multiple sclerosis. Ann Neurol 1987;22:744-52.

9 Rösler KM, Hess CW, Heckmann R, Ludin HP. Significance of shape and size of the stimulating coil in magnetic stimulation of the human motor cortex. Neurosci Lett 1989;100:347-52.

10 Harding AE. Early onset cerebellar ataxia with retained tendon reflexes: a clinical and genetic study of a disorder tendon reflexes: a clinical and genetic study of a disorder
distinct from Friedreich's ataxia. J Neurol Neurosurg distinct from Friedreich's
Psychiatry 1981;44:503-8.

11 Ingram DA, Thompson AJ, Swash M. Central motor conduction in multiple sclerosis: evaluation of abnormalities revealed by transcutaneous magnetic stimulation of the brain. J Neurol Neurosurg Psychiatry 1988;51: 487-94

12 Ingram DA, Swash M. Central motor conduction is abnormal in motor neuron disease. J Neurol Neurosurg Psy chiatry 1987;50:159-66.

13 Schriefer TN, Hess CW, Mills KR, Murray NMF. Central motor conduction studies in motor neurone disease using magnetic brain stimulation. Electroenceph Clin Neurophysiol 1989;74:431-7.

14 Claus D, Harding AE, Hess CW, Mills KR, Murray NMF Thomas PK. Central motor conduction in degenerative ataxic disorders: a magnetic stimulation study. J Neurol ataxic disorders: a magnetic stimulation
Neurosurg Psychiatry 1988;51:790-5.

15 Hess CW, Mills KR, Murray NMF. Central motor conduction in hereditary motor and sensory neuropathy (HMSN). Electroencephalogr Clin Neurophysiol 1987, 66:S46-7.

16 Claus D, Waddy HM, Harding AE, Murray NMF, Thomas PK. Hereditary motor and sensory neuropathies and hereditary spastic paraplegia: a magnetic stimulation study. Ann Neurol 1990;28:43-9. 